\title{
Subject Index to Volume 36
}

A

Achievement, school, 257

Adjustment, social, 239

Age, 467

Altruism, 289

Analysis

genetic, 541

multivariate, 171

pedigree, 21

spectral, 51

Animal models, 433

Apolipoproteins, 439

Ascertainment bias, 67

Autonomous languages, 233

B

Baptism records, 365

Behavior

genetics, 289

sexual, 289

Binary data, 21

Birth

interval, 355

order, 355

size at, 197

Birthweight, 181, 267
Bloodgroup antigens, 417

Blood pressure, 455, 467, 475, 485

Body measurements, 549

Breast cancer, 5

$\mathrm{C}$

Cancer of the breast, 5

Chinese population, 335

Cholesterol, 439

Cognitive development, 181

Constitution, 455

Constrained optimization, 29

Contraceptives, oral, 397, 409

Coronary heart disease, 439

Correlation coefficients, 535

familial, 549

Cotwin control study, 503

D

Damage, neurological, 249

Depressiveness, 257

Design, parent-offspring, 29

Development, 249, 249, 475

cognitive, 181

language, 225 


\section{Subject Index}

mental, 121, 165

personality, 225

Developmental effects, 467

Disease liability, 5

Dominance, 249, 257

Drinking, 493

E

Environment, 455 individual, 467

permissive, 155, 165

restrictive, 155,165

Environment $x$ genotype interaction, 5, 41,145

Environmental factors, 475

Epidemic factors, 373

Epidemiology, 335, 421, 485, 493

Evolution, 289

Exposure, solvent, 503

F

Factors

environmental, 475

epidemic, 373

genetic, 455,475

Familial correlations, 549

Family size, 355

Fecundability, 289

Fecundity, 355

Fertility, 343

Feto-fetal transfusion, 181

G

Genetic

analysis, 541

factors, 455,475

variance, 549

Genetics, 517

behavior, 289
$\mathrm{H}$

Hair whorls, 557

Heart rate, 51

Height, 171, 197, 209

Heredity, 467

Heritability, 171, 523, 535, 549

Higher multiples, 197

Homeotic genes, 433

Hyperlipidemias, 439

Hypertension, 439

I

Immunoglobulins, 509

Inbreeding, 343, 349

India, 549

Intelligence, 249

Interaction

genotype $\mathrm{x}$ environment, $5,41,145$ marital, 41

Iritis, 523

K

Kibbuzz, 155, 165

Kidney, 567

L

Language, 213

acquisition, 233

development, 225

disorders, 233

Languages, autonomous, 233

Left handedness, 421

Life-history analysis, 289

Lifestyle, 485

Linear regression models, 297

LISREL, 29

Logistic regression, 21

Log-linear models, 21

Longitudinal study, $121,135,145,155$, 171,209 
M

Malformations, 421, 433 vascular, 567

Marital interaction, 41 status, 297

Mate selection, 41

Maternal age, 297, 313

Maximum likelihood, 21

Menarche, 541

Menstrual cycle, 541

Mental development, 121, 165

Mental gain scores, 121

Missouri founders, 349

Model fitting procedures, 541

Monochorionic twins, 181, 417

Morbidity, perinatal, 249

Multiple birth rates, 325

Multiples, higher, 197

Multivariate analysis, 171

normality, 21

$\mathrm{N}$

Neurological damage, 249

Neuropathy, 503

O

Optimization, constrained, 29

Ovulation stymulants, 313

$\mathbf{P}$

Parental rating, 135

Parent-offspring design, 29

Parents of twins, 225

Parish records, 355

Parity, 297, 313

Pedigree analysis, 21

Pepsinogen, 517

Perinatal morbidity, 249

Personality, 79

development, 225
Placenta, 417

Play, 213

Prematurity, 239

Prenatal loss, 365

Preschool, 213

Psychosis, 267

Psychosomatic symptoms, 257

Q

Questionnaire, 79

$\mathrm{R}$

Race, 313

Rates

multiple birth, 325

stillbirth, 325

twinning, 297, 313, 325, 335, 343, $355,365,373$

Rating, parental, 135

Registries, 61, 67, 79

Regression, logistic, 21

S

Sampling, 79

School, 155, 239 achievement, 257

Seasonality, 365, 373, 381

Secular trend, 365, 397, 409

Selection, mate, 41

Sex differences, 213

Sexual behavior, 289

Sexual intercourse, 381

Size at birth, 197

Smoking, 493

Sociability, 213

Social adjustment, 239

Solvent exposure, 503

Spectral analysis, 51

Strabismus, 523

Stress disorders, post-traumatic, 67

Substance exposure, 79

Submissiveness, 249,257 


\section{Subject Index}

$\mathrm{T}$

Temperament, 135, 145

Time series, 51

Transfusion, feto-fetal, 181

Twin, 523

births, 381

concordance, 135,535

data, 51

family design, 467

half-sib model, 455

mortality, 275

Twinning, 421, 557

excessive, 349

rates, $297,313,325,335,343,355$,

$365,373,389,397,409$

Twins, 5, 29, 61, 67, 79, 121, 145, 165,

$197,209,213,239,257,289,433$,

$509,517,549,567$

monochorionic, 181,417

MZ, 267, 417 parents of, 225

reared apart, 485,493

U

Uropepsinogen, 517

V

Veterans, 61, 67

Vietnam War, 61, 67

W

Weight, 171, 197, 209

Weinberg's method, 257, 389

Z

Zygosity, 257 